\title{
The Evaluation of Skeletal Manifestations in Patients with Gaucher Disease
}

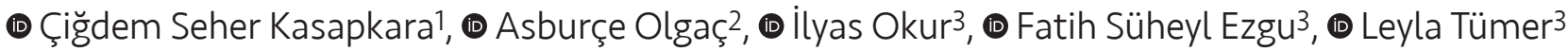 \\ ${ }^{1}$ Ankara Yıldırım Beyazıt University, Ankara City Hospital, Department of Pediatric Metabolism, Ankara, Turkey \\ 2University of Health Sciences Turkey, Dr. Sami Ulus Training and Research Hospital, Department of Pediatric Metabolism, Ankara, Turkey \\ ${ }^{3} \mathrm{Gazi}$ University Hospital, Department of Pediatric Metabolism and Nutrition, Ankara, Turkey
}

\begin{abstract}
Aim: Gaucher disease (GD) is the most prevalent hereditary lysosomal storage disorder, affecting multiple organ systems. It is characterized by a deficiency of the enzyme glucocerebrosidase leading to an accumulation of glucosylceramide in lysosomes. The majority of patients present with hepatosplenomegaly, anemia, thrombocytopenia, bleeding tendencies, skeletal pathologies, growth retardation and in severe cases pulmonary disease. The bone manifestations include bone infarcts, avascular bone necrosis, lytic lesions, osteopenia and osteoporosis. This article gives an overview of the bone manifestations of $20 \mathrm{GD}$ patients and reviews the current literature.
\end{abstract}

Materials and Methods: The data of 20 patients with GD who were being followed up in Gazi University Hospital Pediatric Metabolism Unit were retrospectively evaluated. Their demographic information including age, gender, clinical findings, enzyme replacement therapy (ERT) status and duration were recorded. Laboratory analyses including serum calcium, phosphorus, vitamin $D$, the presence of skeletal findings, and bone mineral density (BMD) Z scores were collected from the patient files. The BMD status of patients was compared with their pre-treatment values.

Results: The main symptoms of referral were abdominal distention, cytopenia, bleeding tendency and skeletal findings. All patients had skeletal symptoms. Nineteen patients showed vitamin D deficiency. The medullary involvement of femur and vertebrae was present in 14 (70\%), Erlenmeyer flask deformity in $3(15 \%)$, and avascular necrosis in one (5\%) patient. Also, one patient (5\%) had lytic bone lesions. Ten patients showed osteoporosis (50\%), and 8 showed osteopenia (40\%) at the time of diagnosis, before the initiation of ERT. The rate of osteoporosis was determined to be $40 \%$, and osteopenia was $35 \%$ within the study group after the initiation of ERT.

Conclusion: Physicians, including pediatricians, may be unfamiliar with bone pathophysiology and the complexity of the skeletal manifestations of GD. There is a need to enhance awareness and to improve the diagnosis and treatment of skeletal pathology in patients with GD.

Keywords: Gaucher disease, skeletal involvement, osteoporosis, osteopenia

\section{Introduction}

Gaucher disease (GD) is an autosomal recessively inherited inborn error of metabolism (IEM) of glycosphingolipids, caused by loss of function mutations in the GBA gene encoding lysosomal glucocerebrosidase, a lysosomal enzyme that is responsible for the breakdown of glucocerebroside. Due to the decreased activity of this enzyme, glucocerebroside accumulates in various tissues, affecting multiple organ systems, and leading to serious complications. GD is subdivided into neuronopathic (types 2 and 3) and non-neuronopathic (type 1) phenotypes. Type 1 is the most prevalent form with an estimated prevalence of 1 in 40,000 to 60,000 cases in the general population (1). 
The skeletal findings associated with GD are osteopenia, osteoporosis, osteonecrosis, osteosclerosis, bone crises, chronic bone pain, Erlenmeyer flask deformity, pathological fractures and vertebral collapse. Vitamin D deficiency is also known to be frequent among patients (2).

Enzyme replacement therapy (ERT) and substrate reducing therapy (SRT) are efficacious in treating the visceral and hematological aspects of the disease, and are also effective in reversing the skeletal findings. Initiating early treatment may prevent skeletal complications which are the most debilitating manifestations of this disease and have a significant impact on the patient's well-being $(1,2)$.

We present the skeletal findings of 20 patients with GD being followed up at Gazi University Hospital, Pediatric Metabolism Unit. Also, an overview of bone manifestations of $G D$, along with a review of the current literature is given.

\section{Materials and Methods}

The data of 20 patients with GD who were being followed up at Gazi University Hospital, Pediatric Metabolism Unit were retrospectively evaluated. All patients had been on ERT for at least six months at the time of the data collection. Their demographic information including age, gender, subtype of GD, age of initial symptoms and diagnosis, clinical findings, age at the initiation of treatment (ERT) and the duration of ERT were recorded. Laboratory analyses including serum calcium (Ca), phosphorus (P), 25-hydroxyvitamin $\mathrm{D}[25(\mathrm{OH}) \mathrm{D}]$, and the presence of skeletal findings were collected from the patient files. The values for vitamin D deficiency and insufficiency were determined according to the criteria of European Society of Paediatric Gastroenterology, Hepatology and Nutrition. Values below $20 \mathrm{ng} / \mathrm{mL}$ (50 nmol/L) were accepted as deficiency and values between 20 and $29.9 \mathrm{ng} / \mathrm{mL}$ (50$74.9 \mathrm{nmol} / \mathrm{L})$ as vitamin $D$ insufficiency. The cut-off for normal vitamin D levels was $\geq 30 \mathrm{ng} / \mathrm{mL}$ ( $\geq 75 \mathrm{nmol} / \mathrm{L}$ ) (3).

Bone mineral density (BMD) measurements were performed as part of routine annual follow-up, and were assessed by dual-energy X-ray absorptiometry at the lumbar vertebrae, hip, and wrist. Values were reported as Z-scores and T-scores, in relation to the deviation in units of Standard deviation from the median age specific and young adult reference values, respectively (4).

The research was approved by the Ethics Committee of Gazi University Medical Faculty (approval no:2020-444, date: 14.07.2020). Written consent was obtained from all subjects.

\section{Statistical Analysis}

Median and ranges were used for descriptive statistics, and paired sample t-test was used to evaluate modifications in mean BMD scores, by using the SPSS 26.0 software package.

\section{Results}

The patient group included seventeen patients with GD type 1, and three patients with type 3 (a total of 20 patients). The mean age of patients was $18.72 \pm 14.71$ years (range: $1-51$ years), the mean age of diagnosis was $14.4 \pm 11.03$ years (range: 1-44 years). The age of initiation of symptoms ranged between 1 to 15 years. The median duration of ERT was $2.52 \pm 2.08$ (range: $0.5-7$ ) years. Of a total of 20 patients, 8 were male and 12 were female. Ten out of 20 patients were diagnosed after the age of 18 years.

All patients had confirmed low levels of glucocerebrosidase enzyme. Additionally, all patients had genetically confirmed variants of the GBA gene. The most common variant detected in GD type 1 patients was the homozygous c.1226A>G variant (10/20 patients), 4 patients had the homozygous c.1448T >C variant. Other detected variants were homozygous c.1214G $>$ A (2/20), compound heterozygous (c.1311-1312insT;c.1226A>G) (1/20) and (c.1226A>G;c.1505G>A) (1/20).

Their main symptoms of referral were abdominal distention (15 cases, 75\%), anemia (2 cases, 10\%), thrombocytopenia (2 cases, 10\%), bleeding tendency (2 cases, $10 \%)$ and skeletal findings (1 case 5\%). One patient was splenectomized (Table I). All patients had visceral findings at the time of the study (100\%).

All patients had skeletal symptoms. Nineteen patients showed vitamin D deficiency. The mean serum concentration of $25(\mathrm{OH}) \mathrm{D}$ was $11.22 \pm 7.92(3-40) \mathrm{ng} / \mathrm{mL}$. The mean $\mathrm{Ca}, \mathrm{P}$ and ALP values were $9.23 \pm 0.39 \mathrm{mg} / \mathrm{dL}$ (8.3-9.9), 3.91 \pm 0.57 $\mathrm{mg} / \mathrm{dL}$ (3-5) and 124.35 $\pm 58.58 \mathrm{IU} / \mathrm{L}$ (48-272) respectively.

Medullary involvement of femur and vertebrae was present in 14 (70\%), Erlenmeyer flask deformity in 3 (15\%), and avascular necrosis in one (5\%) patient. Also, one patient $(5 \%)$ had lytic bone lesions. Ten patients showed osteoporosis (50\%), and 8 showed osteopenia (40\%) at the time of diagnosis, before the initiation of ERT (Table II). Three adult patients claimed to have bone pain (15\%). It was observed that the patient with lytic lesions had been diagnosed at the age of 37, and had been receiving ERT for 3 years.

All of the adult patients showed osteopenia before the initiation of treatment. At the time of the study period, while all patients were on ERT, it was seen that 8 patients had osteoporosis, 7 had osteopenia and 5 showed normal BMD findings. Ten patients showed medullary involvement 
Table I. Baseline general clinical assessment of patients with Gaucher disease

\begin{tabular}{|c|c|}
\hline Gaucher disease subtype & Patients (n) \\
\hline $\begin{array}{l}\text { Type } 1 \\
\text { Type } 3\end{array}$ & $\begin{array}{l}17 \\
3\end{array}$ \\
\hline Gender & Patients (n) \\
\hline $\begin{array}{l}\text { Male } \\
\text { Female }\end{array}$ & $\begin{array}{l}8 \\
12\end{array}$ \\
\hline Mean age $\pm S D$, years (range) & $18.72 \pm 14.71(1-51)$ \\
\hline $\begin{array}{l}\text { Mean age of initation of } \\
\text { symptoms, } \pm \text { SD, years (range) }\end{array}$ & $5.8 \pm 4.89(1-25)$ \\
\hline $\begin{array}{l}\text { Mean age of diagnosis, } \pm S D \text {, } \\
\text { years (range) }\end{array}$ & $14.4 \pm 11.03$ years $(1-44$ \\
\hline Initital symptoms for referral & Patients (n) \\
\hline $\begin{array}{l}\text { Hepatosplenomegaly } \\
\text { (Abdominal distension) } \\
\text { Anemia } \\
\text { Thrombocytopenia } \\
\text { Coagulaopathy } \\
\text { Bone pain }\end{array}$ & $\begin{array}{l}15 \\
2 \\
2 \\
2 \\
1\end{array}$ \\
\hline Splenectomy & 1 \\
\hline Mean ERT duration (years) & $2.52 \pm 2.08(0.5-7)$ \\
\hline $\begin{array}{l}\text { Mean } 25(\mathrm{OH}) \text { D concentration, } \pm \\
\text { SD, (ng/mL) (range) }\end{array}$ & $11.22 \pm 7.92(3-40)$ \\
\hline
\end{tabular}

Table II. Skeletal findings of patients with Gaucher disease

\begin{tabular}{|l|l|l|}
\hline Skeletal manifestation & Baseline (n) & During ERT (n) \\
\hline $\begin{array}{l}\text { Medullary involvement of femur } \\
\text { and vertebrae }\end{array}$ & 14 & 10 \\
\hline Osteoporosis & 10 & 8 \\
\hline Osteopenia & 8 & 7 \\
\hline Bone pain & 3 & 0 \\
\hline Avascular necrosis & 1 & 1 \\
\hline Erlenmeyer flask deformity & 3 & 3 \\
\hline Pathologic fractures & 0 & 0 \\
\hline Bone crisis & 1 & 0 \\
\hline Lytic lesions & 1 & 1 \\
\hline ERT: Enzyme replacement therapy & &
\end{tabular}

of bone and vertebrae. None of the patients had complaints of bone pain, bone crisis or pathological fractures during the ERT period.

\section{Discussion}

$G D$, one of the most common lysosomal storage disorders (LSD), is an IEM of the lysosomal enzyme glucocerebrosidase, which induces the deposition of undegraded glycolipid material in organs rich in mononuclear macrophage system including the liver, spleen and bone marrow. There are three clinical sub-types based on age of onset and the presence of neurological manifestations. GD type 1 is the non-neuronopathic type, with primarily skeletal and visceral signs and symptoms which range in severity. The infantile-onset (type 2) and later-onset (type 3) GD involve the central nervous system. The diagnosis is confirmed by analysis of glucocerebrosidase activity in peripheral blood leukocytes or fibroblasts $(1,2)$.

GD is commonly associated with HSM and occasionally other organs, including the kidneys or lungs, are affected. The skeletal manifestations of CD include a variety of bone pathologies prevalent at all ages, due to the progressive glucocerebroside storage, changes of vascularity, and impaired bone remodeling. Bone involvement is broad and can occur in otherwise clinically asymptomatic individuals (5). Furthermore, bone symptoms may present signs in childhood (6). One of the largest cohorts of GD type 1 patients including 2,004 patients evaluated in the International Collaborative Gaucher Group (ICGG) Gaucher registry showed bone manifestations to be prevalent at a rate of between $76 \%$ and $94 \%$ (7).

The frequently encountered bone manifestations of GD include bone infarcts, bone marrow infiltration, Erlenmeyer flask deformity, avascular necrosis, lytic lesions, osteosclerosis, and fractures due to osteoporosis. The recognition of bone disease related with $G D$ is important since it may lead to serious complications including polyclonal and monoclonal gammopathies and cancer (1-5).

In our study group, the most frequent skeletal manifestation was low BMD including osteopenia and osteoporosis, which was detected in all adult patients before the initiation of ERT. This finding is in accordance with several studies in the literature. Pastores et al. (8) revealed that 61 adult patients with GD had significantly lower BMD than expected for age and sex. The authors stated that the severity of the osteopenia correlated significantly with other clinical indicators, namely disease severity, genotype, prior splenectomy, and hepatomegaly and the severity of skeletal disease as assessed by skeletal radiography. The ICCG Gaucher Registry has indicated that the rate of osteopenia is 55\% among all registry patients $(8,9)$. Mistry et al. (10) showed low bone density to be most prevalent in adolescence. Our findings demonstrate that skeletal findings in GD are mainly encountered after childhood, and since ERT is effective in the regression of skeletal pathologies, early diagnosis and treatment may be 
an important factor in the prevention of irreversible damage to the skeletal system.

ERT is effective in the treatment of GD and can have a significant impact on skeletal manifestations. De Fost et al. (11) have suggested the response to ERT to be slower for the hematological and visceral symptoms, when compared to skeletal symptoms. The efficacy of ERT in reducing the burden of Gaucher cells in the bone marrow has been demonstrated in several studies (12). It has been shown that the Bone Marrow Burden (BMB) score, a radiological scoring system used to evaluate the severity of bone involvement in GD proposed by Maas et al. (13), improves by 2 or more points after ERT (11). Similar beneficial effects have been observed with SRT (14).

It is important to diagnose focal osteolytic lesions of GD, which typically have a "worm-eaten" appearance, since they are a risk factor for fractures, and they may be mistakenly diagnosed as malignancy related osteolytic lesions (15). In our patient group, only one had focal osteolytic lesions along with bone pain and avascular necrosis. Interestingly, this patient was splenectomised before the diagnosis of GD. It has previously been reported that splenectomised patients have lower bone density (8). Thus, the severe skeletal findings of this patient may be related with the splenectomy.

It is well-known that bone complications respond to ERT later than visceral and hematological manifestations. Robertson et al. (12) reported that the BMB score was improved by 2 or more points in those patients receiving ERT. Also, the fact that bone findings stabilize after 5 years of therapy has been previously reported in the literature, reaching near normal values after 7 years of ERT (2). Our findings are in compliance with the literature, since the skeletal findings including lytic lesions were not completely resolved, when the relatively short duration of ERT was considered.

Although vitamin D deficiency is very common among the general population, GD patients have been reported to have a higher prevalence of low vitamin $D$ levels than the general population. Vitamin $D$ deficiency in $G D$ may result from: low intake, malabsorption, diminished exposure to sunlight or decreased hepatic production of calcidiol (16). In our study group, the rate of vitamin D deficiency was found to be $95 \%$. Unfortunately, we were not able to evaluate the etiologic factors contributing to the vitamin $D$ deficiency in our patient group, due to our research being retrospective. Mikosch and Hughes (5) evaluated 74 GD patients and found a high prevalence of vitamin D deficiency among these patients. Similarly, nineteen out of 20 GD patients in our study group had vitamin D deficiency. Considering the essential role of vitamin $\mathrm{D}$ in bone homeostasis and the prevalence of hypovitaminosis $D$, we suggest evaluating this parameter routinely in all GD patients. In order to optimize the care of bone disease in GD patients, supplementation with vitamin D must be recommended for GD patients with osteopenia or osteoporosis.

\section{Conclusion}

BMD normalization was achieved in three patients with ERT. These results suggest that ERT for pediatric and adult patients may be an effective treatment for osteoporosis and osteopenia in these patients and ERT may maintain BMD and lessen the risk of developing osteopenia in patients with near normal BMD scores. We think that since the average duration of ERT was 2.5 years in our study group, the number of patients who benefit from ERT will increase with time, since it is known that bone manifestations stabilize after 5 years of ERT. Also, adequate calcium replacement should be given according to local guidelines.

Physicians, including pediatricians, may be unfamiliar with bone pathophysiology and the complexity of the skeletal manife stations of GD. There is a need to enhance awareness and to improve the diagnosis and treatment of skeletal pathology in patients with GD.

\section{Ethics}

Ethics Committee Approval: The research was approved by the Ethics Committee of Gazi University Medical Faculty (approval no: 2020-444, date: 14.07.2020).

Informed Consent: Written consent was obtained from all subjects.

Peer-review: Externally and internally peer-reviewed.

\section{Authorship Contributions}

Concept: F.S.E., L.T., Design: Ç.S.K., Data Collection or Processing: A.O., Analysis or Interpretation: I.O., Literature Search: Ç.S.K., A.O., I.O., Writing: Ç.S.K., A.O., I.O., F.S.E., L.T.

Conflict of Interest: No conflict of interest was declared by the authors.

Financial Disclosure: The authors declared that this study received no financial support.

\section{References}

1. Mikosch P. Miscellaneous non-inflammatory musculoskeletal conditions. Gaucher disease and bone. Best Pract Res Clin Rheumatol 2011; 25:665-81. 
2. Hughes D, Mikosch P, Belmatoug N, et al. Gaucher disease in bone: from pathophysiology to practice. I Bone Miner Res 2019; 34:996-1013.

3. Braegger C, Campoy C, Colomb V, et al; Vitamin D in the healthy European pediatric population. ESPGHAN Commitee on Nutrition. J Pediatr Gastroenterol Nutr 2013; 56:692-701.

4. Lewiecki EM, Gordon CM, Baim S, et al. International society for clinical densitometry 2007 adult and pediatric official positions. Bone 2008; 43:1115-21.

5. Mikosch P, Hughes D. An overview on bone manifestations in Gaucher disease. Wien Med Wochenschr 2010; 160:609-24.

6. Rossi L, Zulian F, Stirnemann J, Billette de Villemur T, Belmatoug $\mathrm{N}$. Bone involvement as presenting sign of pediatric-onset Gaucher disease. Joint Bone Spine 2011; 78:70-4.

7. Wenstrup RJ, Roca-Espiau M, Weinreb NJ, Bembi B. Skeletal aspects of Gaucher disease: a review. Br I Radiol 2002; 75(Suppl 1):A2-12. doi: 10.1259/bjr.75.suppl 1.750002.

8. Pastores GM, Wallenstein S, Desnick RJ, Luckey MM. Bone density in Type 1 Gaucher disease. J Bone Miner Res 1996; 11:18017.

9. Charrow J, Andersson HC, Kaplan P, et al. The Gaucher registry: demographics and disease characteristics of 1698 patients with Gaucher disease. Arch Intern Med 2000; 160:2835-43.

10. Mistry PK, Weinreb NJ, Kaplan P, et al. Osteopenia in Gaucher disease develops early in life: response to imiglucerase enzyme therapy in children, adolescents and adults. Blood Cells Mol Dis 2011; 46:66-72.

11. De Fost M, Hollak CEM, Groener JEM, et al. Superior effects of high-dose enzyme replacement therapy in type 1 Gaucher disease on bone marrow involvement and chitotriosidase levels: a 2- center retrospective analysis. Blood 2006; 108:830-5.

12. Robertson PL, Maas M, Goldblatt J. Semiquantitative assessment of skeletal response to enzyme replacement therapy for Gaucher's disease using the bone marrow burden score. AJR Am / Roentgenol 2007; 188:1521-8.

13. Maas M, van Kujik C, Stoker J, et al. Quantification of bone involvement in Gaucher disease: MR imaging bone marrow burden score as an alternative to Dixon quantitative chemical shift MR imaging-initial experience. Radiology 2003; 229:554-61.

14. Lukina E, Watman N, Dragosky M, et al. Outcomes after 8 years of eliglustat therapy for Gaucher disease type 1: final results from the Phase 2 trial. Am J Hematol 2019; 94:29-38.

15. Khan A, Hangartner T, Weinreb NJ, Taylor JS, Mistry PK. Risk factors for fractures and avascular osteonecrosis in type 1 Gaucher disease: a study from the International Collaborative Gaucher Group (ICGG) Gaucher Registry I Bone Miner Res 2012; 27:1839-48.

16. Mikosch $P$, Reed $M$, Stettner $H$, Baker $R$, Mehta $A B$, Hughes DA. Patients with Gaucher disease living in England show a high prevalence of vitamin D insufficiency with correlation to osteodensitometry. Mol Genet Metab 2009; 96:113-20. 